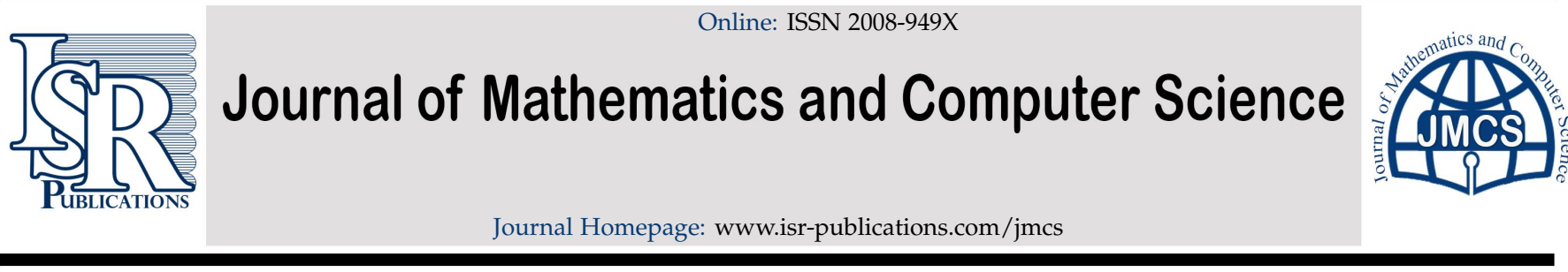

\title{
The r-Bell numbers and matrices containing non-central Stir- ling and Lah numbers
}

\author{
Roberto B. Corcino*, Cristina B. Corcino, Jeneveb T. Malusay, Gaea lolanthe Mari R. Bercero
}

Research Institute for Computational Mathematics and Physics, Cebu Normal University, Osmeña Boulevard, Cebu City, Philippines.

\begin{abstract}
In this paper, two new explicit formulas for $r$-Bell numbers are established. One formula is expressed in terms of $r$-Stirling numbers of the second kind and r-Lah numbers. The other formula is expressed in terms of the non-central Stirling numbers of the second kind and the ordinary Lah numbers. Moreover, some matrix relations are obtained involving $r$-Bell numbers, r-Stirling numbers of the second kind, r-Lah numbers, non-central Stirling numbers of the second kind, and the ordinary Lah numbers.
\end{abstract}

Keywords: r-Bell numbers, r-Stirling numbers, non-central Stirling numbers, $r$-Lah numbers, Lah numbers.

2010 MSC: 05A15, 11B65, 11B73.

(C)2019 All rights reserved.

\section{Introduction}

In a recent paper by Qi [9], the Bell numbers were given explicit formula expressed in terms of Lah and Stirling numbers of the second kind as follows:

$$
B_{n}=\sum_{k=1}^{n}(-1)^{n-k}\left[\sum_{l=1}^{k} L(k, l)\right] S(n, k),
$$

where $L(k, l)$ denote the Lah numbers and $S(n, k)$ the Stirling numbers of the second kind. These numbers can be interpreted in terms of set partition as follows:

$S(n, k):=$ the number of partitions of an $n$-set into $k$ nonempty subsets;

$\mathrm{L}(\mathrm{k}, \mathrm{l}):=$ the number of partitions of an $n$-set into $\mathrm{k}$ nonempty linearly ordered subsets;

$\mathrm{B}_{\mathrm{n}}:=$ the number of partitions of an $n$-set.

\footnotetext{
${ }^{*}$ Corresponding author

Email addresses: rcorcino@yahoo.com (Roberto B. Corcino), cristinacorcino@yahoo.com (Cristina B. Corcino), ferdiemalusay@yahoo.com (Jeneveb T. Malusay), gimbercero@gmail.com (Gaea Iolanthe Mari R. Bercero)

doi: $10.22436 /$ jmcs.019.03.05
}

Received: 2019-04-15 Revised: 2019-04-25 Accepted: 2019-05-02 
Now, consider two $n \times n$ matrices $[\hat{S}(i, j)]_{n \times n}$ and $[L(i, j)]_{n \times n}$ whose entries are respectively the Stirling numbers of the second kind and the Lah numbers. More precisely, we have

$$
\begin{aligned}
{[\hat{S}(i, j)]_{n \times n} } & =\left[\begin{array}{ccccc}
\hat{S}(1,1) & 0 & 0 & \ldots & 0 \\
\hat{S}(2,1) & \hat{S}(2,2) & 0 & \ldots & 0 \\
& \ldots \ldots \ldots \ldots \ldots \ldots \ldots & \\
\hat{S}(n, 1) & \hat{S}(n, 2) & \hat{S}(n, 3) & \ldots & \hat{S}(n, n)
\end{array}\right], \\
{[L(i, j)]_{n \times n} } & =\left[\begin{array}{ccccc}
L(1,1) & 0 & 0 & \ldots & 0 \\
L(2,1) & L(2,2) & 0 & \ldots & 0 \\
& \ldots \ldots \ldots \ldots \ldots \ldots & \\
L(n, 1) & L(n, 2) & L(n, 3) & \ldots & L(n, n)
\end{array}\right],
\end{aligned}
$$

where $\hat{S}(i, j)=(-1)^{i-j} S(i, j)$. It can easily be seen using the explicit formula in (1.1) that the Bell numbers $B_{i}$ equal to the sum of the entries of the $i^{\text {th }}$ row of the matrix $\left[p_{i, j}\right]_{n \times n^{\prime}}$, where

$$
\left[p_{i, j}\right]_{n \times n}=[\hat{S}(i, j)]_{n \times n}[L(i, j)]_{n \times n} \text {. }
$$

The $r$-Stirling numbers of the first and second kind, denoted by $s(n, k ; r)$ and $S(n, k ; r)$, are respectively defined by Broder [2] as follows:

$s(n, k ; r):=$ the number of permutations of an $n$-set into $k$ nonempty cycles such that the numbers $1,2, \ldots, r$ are in distinct cycles;

$S(n, k ; r):=$ the number of partitions of an $n$-set into $k$ nonempty subsets such that the numbers $1,2, \ldots, r$ are in distinct subsets.

These numbers have possessed several properties including recurrence relations, explicit formulas, generating functions and the following inverse relations

$$
b_{n}=\sum_{k=0}^{n} s(n, k ; r) a_{k} \Longleftrightarrow a_{n}=\sum_{k=0}^{n}(-1)^{n-k} S(n, k ; r) b_{k}
$$

and the relation containing the classical Stirling numbers of the second kind

$$
S(n, k ; r)=\sum_{j=k}^{n}\left(\begin{array}{l}
n \\
j
\end{array}\right) S(j, k) r^{n-j}
$$

It is worth mentioning that the r-Stirling numbers of the second kind are closely related to the non-central Stirling numbers of the second kind. Koutras [6] has defined the non-central Stirling numbers of the second kind, denoted by $S_{a}(n, k)$, by means of the following $k^{\text {th }}$ differences

$$
S_{a}(n, k)=\frac{1}{k !}\left[\Delta^{k}(t-a)^{n}\right]_{t=0},
$$

where $a$ and $t$ are real numbers and $n, k$ are non-negative integers. The non-central Stirling numbers of the second kind have possessed several properties including their relation with Stirling numbers of the second kind

$$
S_{a}(n, k)=\sum_{j=k}^{n}\left(\begin{array}{l}
n \\
j
\end{array}\right) S(j, k)(-a)^{n-j},
$$

the explicit formula

$$
S_{a}(n, k)=\frac{1}{k !} \sum_{j=0}^{k}(-1)^{k-j}\left(\begin{array}{l}
k \\
j
\end{array}\right)(j-a)^{n},
$$


the triangular recurrence relation

$$
S_{a}(n+1, k)=S_{a}(n, k-1)+(k-a) S_{a}(n, k),
$$

and the horizontal generating function

$$
(t-a)^{n}=\sum_{k=0}^{n} S_{a}(n, k)(t)_{k} .
$$

Clearly, for any non-negative integer $r$, the non-central Stirling numbers of the second kind can be expressed in terms r-Stirling numbers of the second kind with negative index as follows:

$$
S_{r}(n, k)=S(n, k ;-r) .
$$

For example, the values of $r$-Stirling numbers of the second kind when $n=3, r=-3$ are given by

$$
\begin{aligned}
& S(2,0 ;-2)=S_{2}(2,0)=\frac{1}{0 !}(-2)^{2}=4, \\
& S(2,1 ;-2)=S_{2}(2,1)=\frac{1}{1 !}(-4+1)=-3, \\
& S(2,2 ;-2)=S_{2}(2,2)=\frac{1}{2 !}(4+(-1)(2)(1)+0)=1 .
\end{aligned}
$$

It has been mentioned in [6] that these numbers appear in the distribution of the sum $W=X+Y$ where $X$ is the sum of $k$ independent random variables following the the truncated Poisson distribution away from zero and $Y$ is a Poisson random variable.

The r-Bell numbers, denoted by $B_{n, r}$, were defined by Mezo [7] as the sum of $r$-Stirling numbers of the second kind. More precisely,

$$
B_{n, r}=\sum_{k=0}^{n} S(n+r, k+r ; r) .
$$

Obviously, the $r$-Bell numbers $B_{n, r}$ can be interpreted as the number of partitions of a set with $n+r$ elements such that the first $r$ elements are in distinct subsets in each partition.

Mezo [7] have obtained several properties for $r$-Bell numbers and one of these is the exponential generating function, which is given by

$$
\sum_{n=0}^{\infty} B_{n, r} \frac{z^{n}}{n !}=e^{e^{z}-1+r z} .
$$

Clearly, when $r=0$, this will give the exponential generating function for the ordinary Bell numbers.

On the other hand, Nyul and Racz [8] defined combinatorially the r-Lah numbers $L(n, k ; r)$ as follows:

$L(n, k ; r):=$ the number of partitions of a set with $n+r$ elements into $k+r$ nonempty ordered subsets such that $r$ distinguished elements have to be in distinct ordered blocks.

In particular, when $r=0$, these numbers will reduce to the ordinary Lah numbers. That is,

$$
\mathrm{L}(\mathrm{n}, \mathrm{k} ; 0)=\mathrm{L}(\mathrm{n}, \mathrm{k}) \text {. }
$$

The $r$-Lah numbers have also possessed several properties including their relation with $r$-Stirling numbers of the first and second kind

$$
L(n, k ; r)=\sum_{j=k}^{n} s(n, j ; r) S(j, k ; r),
$$


the orthogonality relation

$$
\sum_{j=k}^{n}(-1)^{n-j} L(n, j ; r) L(j, k ; r)=\delta_{n, k}
$$

and the inverse relation

$$
b_{n}=\sum_{k=0}^{n} L(n, k ; r) a_{k} \Longleftrightarrow a_{n}=\sum_{k=0}^{n}(-1)^{n-k} L(n, k ; r) b_{k} .
$$

In this present paper, we establish a relation between $r$-Bell numbers and the sums of row entries of the product of two matrices containing $r$-Lah numbers and r-Stirling numbers of the second kind. Moreover, as consequences of this relation, some interesting matrix relations are obtained involving the r-Stirling numbers of the first kind. Finally, another form of matrix relation is established between a matrix whose sums of row entries are r-Bell numbers and the product of two matrices whose entries are non-central Stirling numbers of the second kind and Lah numbers. As consequences of this relation, some interesting matrix relations are obtained involving the non-central Stirling numbers of the first kind.

\section{Preliminary results}

The inverse relation in (1.3) can be written as

$$
b_{n}=\sum_{j=0}^{n} s(n, j ; r) a_{j} \Longleftrightarrow a_{n}=\sum_{j=0}^{n}(-1)^{n-j} s(n, j ; r) b_{j} .
$$

Using (1.7) with $b_{n}=L(n, k ; r)$ and $a_{j}=S(j, k ; r)$, we have

$$
\begin{aligned}
a_{n} & =\sum_{j=0}^{n}(-1)^{n-j} S(n, j ; r) b_{j}, \\
S(n, k ; r) & =\sum_{j=0}^{n}(-1)^{n-j} S(n, j ; r) L(j, k ; r) .
\end{aligned}
$$

This relation implies the following matrix equation

$$
[S(i, j ; r)]_{n \times n}=\left[(-1)^{i-j} S(i, j ; r)\right]_{n \times n}[S(i, j ; r)]_{n \times n} .
$$

To illustrate this equation, let us consider the case where $r=5$ and $n=6$. That is,

$$
\begin{aligned}
{\left[(-1)^{i-j} S(i, j ; 5)\right]_{6 \times 6}[L(i, j ; 5)]_{6 \times 6} } & \\
= & {\left[\begin{array}{cccccc}
1 & 0 & 0 & 0 & 0 & 0 \\
-5 & 1 & 0 & 0 & 0 & 0 \\
25 & -11 & 1 & 0 & 0 & 0 \\
-125 & 91 & -18 & 1 & 0 & 0 \\
625 & -671 & 217 & -26 & 1 & 0 \\
-3125 & 4651 & -2190 & 425 & -35 & 1
\end{array}\right]\left[\begin{array}{cccccc}
1 & 0 & 0 & 0 & 0 & 0 \\
10 & 1 & 0 & 0 & 0 & 0 \\
110 & 22 & 1 & 0 & 0 & 0 \\
1320 & 396 & 36 & 1 & 0 & 0 \\
17160 & 6864 & 936 & 52 & 1 & 0 \\
240240 & 120120 & 21840 & 1820 & 70 & 1
\end{array}\right] } \\
= & {\left[\begin{array}{cccccc}
1 & 0 & 0 & 0 & 0 & 0 \\
5 & 1 & 0 & 0 & 0 & 0 \\
25 & 11 & 1 & 0 & 0 & 0 \\
125 & 91 & 18 & 1 & 0 & 0 \\
625 & 671 & 217 & 26 & 1 & 0 \\
3125 & 4651 & 2190 & 425 & 35 & 1
\end{array}\right]=[S(i, j ; 5)]_{6 \times 6} . }
\end{aligned}
$$


Furthermore, replacing $k$ with $j$ and summing up both sides over $i$ of the equation in (2.1) yield

$$
\begin{aligned}
S(n, j ; r) & =\sum_{k=0}^{n}(-1)^{n-k} S(n, k ; r) L(k, j ; r), \\
\sum_{j=0}^{n} S(n, j ; r) & =\sum_{j=0}^{n} \sum_{k=0}^{n}(-1)^{n-k} S(n, k ; r) L(k, j ; r), \\
B_{n, r} & =\sum_{k=0}^{n}(-1)^{n-k}\left[\sum_{j=0}^{k} L(k, j ; r)\right] S(n, k ; r) .
\end{aligned}
$$

This is exactly the explicit formula that appeared in [4]. Now, we can rewrite the above sum as

$$
\mathrm{B}_{\mathrm{n}, \mathrm{r}}=\mathrm{S}_{1}+\mathrm{S}_{2}+\cdots+\mathrm{S}_{\mathrm{n}}
$$

where

$$
S_{j}=\sum_{k=0}^{n}(-1)^{n-k} S(n, k ; r) L(k, j ; r), j=1,2, \ldots, n .
$$

This result is formally stated in the following theorem.

Theorem 2.1. For $n \in \mathbb{N}$, the r-Bell numbers $B_{i, r}$ equal to the sum of the entries of the $i^{\text {th }}$ row of the product of two matrices

$$
\left[(-1)^{i-j} S(i, j ; r)\right]_{n \times n}[L(i, j ; r)]_{n \times n},
$$

whose entries are respectively the r-Stirling numbers of the second kind and the r-Lah numbers.

For example, if we sum up the entries of each row of the matrix in (2.3), we obtain the column vector whose entries are the r-Bell numbers

$$
\left[\begin{array}{c}
1 \\
5+1 \\
25+11+1 \\
125+91+18+1 \\
625+671+217+26+1 \\
3125+4651+2190+425+35+1
\end{array}\right]=\left[\begin{array}{c}
1 \\
6 \\
37 \\
235 \\
1540 \\
10427
\end{array}\right]=\left[\begin{array}{c}
\mathrm{B}_{0,5} \\
\mathrm{~B}_{1,5} \\
\mathrm{~B}_{2,5} \\
\mathrm{~B}_{3,5} \\
\mathrm{~B}_{4,5} \\
\mathrm{~B}_{5,5}
\end{array}\right] .
$$

Now, let us consider some interesting consequences of the matrix relation in (2.2). First, we recall that the r-Stirling numbers of the first and second kind satisfy the following orthogonality relation [2]

$$
\sum_{k} s(k, n ; r) S(m, k ; r)(-1)^{k}=(-1)^{n} \delta_{m, n}
$$

When $\mathrm{m}=\mathrm{n}$, this can be written as

$$
\sum_{k=0}^{n}(-1)^{n-k} S(n, k ; r) L(k, n ; r)=\delta_{n, n}=1,
$$

and when $m \neq n, \delta_{m, n}=0$, which gives

$$
\sum_{k}(-1)^{m-k} S(m, k ; r) s(k, n ; r)=(-1)^{n-m} \delta_{m, n}=0 .
$$

Clearly, equations (2.4) and (2.5) imply the following matrix relation

$$
\left[(-1)^{i-j} S(i, j ; r)\right]_{n \times n}[s(i, j ; r)]_{n \times n}=I_{n},
$$


where $I_{n}$ is the identity matrix of order $n$. Thus,

$$
\left[(-1)^{i-j} S(i, j ; r)\right]_{n \times n}^{-1}=[s(i, j ; r)]_{n \times n} .
$$

Multiplying both sides of the matrix relation in (2.2) by

$$
\left[(-1)^{i-j} S(i, j ; r)\right]_{n \times n}^{-1}
$$

yields

$$
[s(i, j ; r)]_{n \times n}[s(i, j ; r)]_{n \times n}=[S(i, j ; r)]_{n \times n},
$$

which is equivalent to the identity in (1.7). To illustrate equation (2.6), let us consider the case where $r=5$ and $n=6$. That is,

$$
\begin{aligned}
{[s(i, j ; 5)]_{6 \times 6}[S(i, j ; 5)]_{6 \times 6} } & =\left[\begin{array}{cccccc}
1 & 0 & 0 & 0 & 0 & 0 \\
5 & 1 & 0 & 0 & 0 & 0 \\
30 & 11 & 1 & 0 & 0 & 0 \\
210 & 107 & 18 & 1 & 0 & 0 \\
1680 & 1066 & 251 & 26 & 1 & 0 \\
15120 & 11274 & 3325 & 485 & 35 & 1
\end{array}\right]\left[\begin{array}{cccccc}
1 & 0 & 0 & 0 & 0 & 0 \\
5 & 1 & 0 & 0 & 0 & 0 \\
25 & 11 & 1 & 0 & 0 & 0 \\
125 & 91 & 18 & 1 & 0 & 0 \\
625 & 671 & 217 & 26 & 1 & 0 \\
3125 & 4651 & 2190 & 425 & 35 & 1
\end{array}\right] \\
= & {\left[\begin{array}{cccccc}
1 & 0 & 0 & 0 & 0 & 0 \\
10 & 1 & 0 & 0 & 0 & 0 \\
110 & 22 & 1 & 0 & 0 & 0 \\
1320 & 396 & 36 & 1 & 0 & 0 \\
17160 & 6864 & 936 & 52 & 1 & 0 \\
240240 & 120120 & 21840 & 1820 & 70 & 1
\end{array}\right]=[\mathrm{L}(i, j ; 5)]_{6 \times 6} . }
\end{aligned}
$$

Remark 2.2. One can easily see that equation (1.8) implies

$$
[L(i, j ; r)]_{n \times n}^{-1}=\left[(-1)^{i-j} S(i, j ; r)\right]_{n \times n} .
$$

Hence, the matrix relation in (2.6) can further be written as

$$
\left[(-1)^{i-j} L(i, j ; r)\right]_{n \times n}[s(i, j ; r)]_{n \times n}[S(i, j ; r)]_{n \times n}=I_{n} .
$$

\section{Main results}

Parallel to the method of Qi [9], one can derive another form of explicit formula for r-Bell numbers using the Faa di Bruno's formula and certain identity of Bell polynomials of the second kind. The following theorem contains the desired formula, which is expressed in terms of the non-central Stirling numbers of the second kind and the ordinary Lah numbers.

Theorem 3.1. For $\mathrm{n} \in \mathbb{N}$, the $\mathrm{r}$-Bell numbers $\mathrm{B}_{\mathrm{n}, \mathrm{r}}$ are equal to

$$
B_{n, r}=\sum_{k=0}^{n}(-1)^{n-k}\left\{\sum_{j=1}^{k} L(k, j)\right\} S_{r}(n, k) .
$$

Proof. Let us recall the following identity from $[1,5]$ on the $n^{\text {th }}$ derivative of the exponential function $e^{ \pm \frac{1}{t}}$ expressed in terms of the Lah numbers

$$
\left(e^{ \pm \frac{1}{t}}\right)^{(n)}=(-1)^{n} e^{ \pm \frac{1}{t}} \sum_{k=1}^{n}( \pm 1)^{k} L(n, k) \frac{1}{t^{n+k}}
$$


the identity from [3] on Bell polynomials of the second kind

$$
B_{n, k}\left(a b x_{1}, a b^{2} x_{2}, \ldots, a b^{n-k+1} x_{n-k+1}\right)=a^{k} b^{n} B_{n, k}\left(x_{1}, x_{2}, \ldots, x_{n-k+1}\right),
$$

and the famous identity from [3] on Faá di Bruno formula described in terms of the Bell polynomials of the second kind

$$
\frac{d^{n}}{d t^{n}} f \circ h(t)=\sum_{k=0}^{n} f^{(k)}(h(t)) B_{n, k}\left(h^{\prime}(t), h^{\prime \prime}(t), \ldots, h^{(n-k+1)}(t)\right) .
$$

Then taking $k^{\text {th }}$ derivative both sides of (1.6) with $z$ replaced by $-z$ yields

$$
e \sum_{n=k}^{\infty}(-1)^{k} B_{n, r} \frac{z^{n-k}}{(n-k) !}=\frac{d^{k}}{d z^{k}} e^{e^{-z}-r z}
$$

Using Leibniz formula, we have

$$
\begin{aligned}
\frac{d^{n}}{d z^{n}} e^{e^{-z}-r z} & =\frac{d^{n}}{d z^{n}} e^{e^{-z}} e^{-r z} \\
& =\sum_{k=0}^{n}\left(\begin{array}{l}
n \\
k
\end{array}\right) \frac{d^{k}}{d z^{k}} e^{e^{-z}} \frac{d^{n-k}}{d z^{n-k}} e^{-r z} \\
& =\sum_{k=0}^{n}\left(\begin{array}{l}
n \\
k
\end{array}\right)\left\{\sum_{j=0}^{k} \frac{d^{j}}{d u^{j}} e^{1 / u(z)} B_{k, j}\left(u^{\prime}(z), u^{\prime \prime}(z), \ldots, u^{(k-j+1)}(z)\right)\right\} \times(-r)^{n-k} e^{-r z},
\end{aligned}
$$

where $u(z)=e^{z}$. When $k=n$ and evaluating at $z=0$, equation (3.2) gives

$$
\begin{aligned}
e(-1)^{n} B_{n, r} & =e \sum_{k=0}^{n}\left(\begin{array}{l}
n \\
k
\end{array}\right) \sum_{j=0}^{k}\left\{(-1)^{j} \sum_{l=1}^{j} L(j, l)\right\} S(k, j)(-r)^{n-k}, \\
B_{n, r} & =\sum_{j=0}^{n} \sum_{l=0}^{j}\left\{\sum_{k=j}^{n}\left(\begin{array}{l}
n \\
k
\end{array}\right) S(k, j)(-r)^{n-k}\right\}(-1)^{n-j} L(j, l) .
\end{aligned}
$$

Applying the above property of $r$-Stirling numbers of the second kind in (1.4) yields

$$
B_{n, r}=\sum_{j=0}^{n} \sum_{l=0}^{j}\left\{\sum_{k=j}^{n}\left(\begin{array}{l}
n \\
k
\end{array}\right) S(k, j)(-r)^{n-k}\right\}(-1)^{n-j} L(j, l)=\sum_{j=0}^{n}(-1)^{n-j} S_{r}(n, k) \sum_{l=0}^{j} L(j, l) .
$$

This is exactly the formula in (3.1).

The following corollary is a direct consequence of Theorem 3.1.

Corollary 3.2. For $n \in \mathbb{N}$, the $r$-Bell numbers $B_{i, r}$ equal to the sum of the entries of the $i^{\text {th }}$ row of the product of two matrices

$$
\left[(-1)^{i-j} S_{r}(i, j)\right]_{n \times n}[L(i, j)]_{n \times n},
$$

whose entries are respectively the non-central Stirling numbers of the second kind and the Lah numbers.

Proof. We can rewrite the formula in Theorem 3.1 as

$$
B_{i, r}=\sum_{l=0}^{i} T_{i l}, \quad i=0,1,2, \ldots, n,
$$


where

$$
\mathrm{T}_{i l}=\sum_{j=0}^{i}(-1)^{i-j} S_{r}(i, j) L(j, l), \quad l=0,1,2, \ldots, i .
$$

Clearly, $T_{i l}$ is the $(i, l)$-entry of the product of two matrices as $\left[(-1)^{i-j} S_{r}(i, j)\right]_{n \times n}[L(i, j)]_{n \times n}$, containing the non-central Stirling Numbers of the second kind and Lah numbers, respectively.

To illustrate this corollary, let us consider the case where $r=2, n=6$. That is,

$$
\begin{aligned}
{\left[(-1)^{i-j} S_{2}(i, j)\right]_{6 \times 6}[L(i, j)]_{6 \times 6} } & =\left[\begin{array}{cccccc}
1 & 0 & 0 & 0 & 0 & 0 \\
2 & 1 & 0 & 0 & 0 & 0 \\
4 & 3 & 1 & 0 & 0 & 0 \\
8 & 7 & 3 & 1 & 0 & 0 \\
16 & 15 & 7 & 2 & 1 & 0 \\
32 & 31 & 15 & 5 & 0 & 1
\end{array}\right]\left[\begin{array}{cccccc}
1 & 0 & 0 & 0 & 0 & 0 \\
0 & 1 & 0 & 0 & 0 & 0 \\
0 & 2 & 1 & 0 & 0 & 0 \\
0 & 6 & 6 & 1 & 0 & 0 \\
0 & 24 & 36 & 12 & 1 & 0 \\
0 & 120 & 240 & 120 & 20 & 1
\end{array}\right] \\
& =\left[\begin{array}{cccccc}
1 & 0 & 0 & 0 & 0 & 0 \\
2 & 1 & 0 & 0 & 0 & 0 \\
4 & 5 & 1 & 0 & 0 & 0 \\
8 & 19 & 9 & 1 & 0 & 0 \\
16 & 65 & 55 & 14 & 1 & 0 \\
32 & 211 & 285 & 125 & 20 & 1
\end{array}\right] .
\end{aligned}
$$

Hence, summing up the entries of each row of the matrix in (3.3) gives the following column vector whose entries are the $r$-Bell numbers with $r=2$

$$
\left[\begin{array}{c}
1 \\
2+1 \\
4+5+1 \\
8+19+9+1 \\
16+65+55+14+1 \\
32+211+285+125+20+1
\end{array}\right]=\left[\begin{array}{c}
1 \\
3 \\
10 \\
37 \\
151 \\
674
\end{array}\right]=\left[\begin{array}{c}
\mathrm{B}_{0,2} \\
\mathrm{~B}_{1,2} \\
\mathrm{~B}_{2,2} \\
\mathrm{~B}_{3,2} \\
\mathrm{~B}_{4,2} \\
\mathrm{~B}_{5,2}
\end{array}\right] .
$$

We observe that the entries of the matrix in (3.3) are values of r-Stirling numbers of the second kind $S(i, j ; 2)$ when $i, l=0,1, \ldots, 6$. That is,

$$
\left[\begin{array}{cccccc}
1 & 0 & 0 & 0 & 0 & 0 \\
2 & 1 & 0 & 0 & 0 & 0 \\
4 & 5 & 1 & 0 & 0 & 0 \\
8 & 19 & 9 & 1 & 0 & 0 \\
16 & 65 & 55 & 14 & 1 & 0 \\
32 & 211 & 285 & 125 & 20 & 1
\end{array}\right]=[S(i, j ; 2)]_{6 \times 6}
$$

This implies that $S(i, j ; r)=\sum_{j=0}^{i}(-1)^{i-j} S_{2}(i, j) L(j, l), i, l=0,1, \ldots, 6$.

Remark 3.3. In general, the r-Stirling numbers of the second kind satisfy the following explicit formula

$$
S(i, j ; r)=\sum_{j=0}^{i}(-1)^{i-j} S_{r}(i, j) L(j, l),
$$

that is,

$$
[S(i, j ; r)]_{n \times n}=\left[(-1)^{i-j} S_{r}(i, j)\right]_{n \times n}[L(i, j)]_{n \times n}
$$


For example,

$$
\begin{aligned}
{\left[(-1)^{i-j} S_{5}(i, j)\right]_{6 \times 6}[L(i, j)]_{6 \times 6} } & =\left[\begin{array}{cccccc}
1 & 0 & 0 & 0 & 0 & 0 \\
5 & 1 & 0 & 0 & 0 & 0 \\
25 & 9 & 1 & 0 & 0 & 0 \\
125 & 61 & 12 & 1 & 0 & 0 \\
625 & 369 & 97 & 14 & 1 & 0 \\
3125 & 2101 & 660 & 125 & 15 & 1
\end{array}\right]\left[\begin{array}{cccccc}
1 & 0 & 0 & 0 & 0 & 0 \\
0 & 1 & 0 & 0 & 0 & 0 \\
0 & 2 & 1 & 0 & 0 & 0 \\
0 & 6 & 6 & 1 & 0 & 0 \\
0 & 24 & 36 & 12 & 1 & 0 \\
0 & 120 & 240 & 120 & 20 & 1
\end{array}\right] \\
= & {\left[\begin{array}{cccccc}
1 & 0 & 0 & 0 & 0 & 0 \\
5 & 1 & 0 & 0 & 0 & 0 \\
25 & 11 & 1 & 0 & 0 & 0 \\
125 & 91 & 18 & 1 & 0 & 0 \\
625 & 671 & 217 & 26 & 1 & 0 \\
3125 & 4651 & 2190 & 425 & 35 & 1
\end{array}\right]=[S(i, j ; 5)]_{6 \times 6} . }
\end{aligned}
$$

This is exactly the matrix relation in (3.3) when $r=5, n=6$.

Theorem 3.4. For $n \in \mathbb{N}$, the following matrix relation holds

$$
\left[(-1)^{i-j} s_{r}(i, j)\right]_{n \times n}[S(i, j ; r)]_{n \times n}=[L(i, j)]_{n \times n},
$$

which is equivalent to the following identity:

$$
L(n, j)=\sum_{k=0}^{n}(-1)^{n-k} s_{r}(n, k) S(k, j ; r) .
$$

Proof. We recall that the non-central Stirling numbers of the first kind, denoted by $s_{a}(n, k)$, satisfy the following horizontal generating function

$$
(t)_{n}=\sum_{k=0}^{n} s_{r}(n, k)(t-r)^{k}
$$

We can rewrite equations (1.5) and (3.5) as follows:

$$
(t+r)^{n}=\sum_{k=0}^{n}(-1)^{n} S_{r}(n, k)(-t)_{k}, \quad(-t)_{n}=\sum_{k=0}^{n}(-1)^{k} s_{r}(n, k)(t+r)_{k} .
$$

These imply the following orthogonality relation

$$
\sum_{k=0}^{n}(-1)^{n-j} S_{r}(n, k) s_{r}(k, j)=\delta_{n, j}
$$

which can further be written as

$$
\sum_{k=0}^{n}\left[(-1)^{n-k} S_{r}(n, k)\right]\left[(-1)^{k-j} s_{r}(k, j)\right]=\delta_{n, j} .
$$

Equation (3.6) gives the following matrix relation

$$
\left[(-1)^{i-j} S_{r}(i, j)\right]_{n \times n}\left[(-1)^{i-j} S_{r}(i, j)\right]_{n \times n}=I_{n},
$$

where $I_{n}$ is the identity matrix of order $n$. Thus,

$$
\left[(-1)^{i-j} S_{r}(i, j)\right]_{n \times n}^{-1}=\left[(-1)^{i-j} s_{r}(i, j)\right]_{n \times n} .
$$


Multiplying both sides of the matrix relation in Remark 3.3 by $\left[(-1)^{i-j} S_{r}(i, j)\right]_{n \times n}^{-1}$, yields

$$
\left[(-1)^{i-j} S_{r}(i, j)\right]_{n \times n}^{-1}[S(i, j ; r)]_{n \times n}=[L(i, j)]_{n \times n},
$$

which is equivalent to (3.4).

For example, when $n=5$ and $r=5$, we have

$$
\begin{aligned}
{\left[(-1)^{i-j} s_{5}(i, j)\right]_{6 \times 6}[S(i, j ; 5)]_{6 \times 6} } & =\left[\begin{array}{ccccccc}
1 & 0 & 0 & 0 & 0 & 0 \\
-5 & 1 & 0 & 0 & 0 & 0 \\
20 & -9 & 1 & 0 & 0 & 0 \\
-60 & 47 & -12 & 1 & 0 & 0 \\
120 & -154 & 71 & -14 & 1 & 0 \\
-120 & 274 & -225 & 85 & -15 & 1
\end{array}\right]\left[\begin{array}{cccccc}
1 & 0 & 0 & 0 & 0 & 0 \\
5 & 1 & 0 & 0 & 0 & 0 \\
25 & 11 & 1 & 0 & 0 & 0 \\
125 & 91 & 18 & 1 & 0 & 0 \\
625 & 671 & 217 & 26 & 1 & 0 \\
3125 & 4651 & 2190 & 425 & 35 & 1
\end{array}\right] \\
& =\left[\begin{array}{cccccc}
1 & 0 & 0 & 0 & 0 & 0 \\
0 & 1 & 0 & 0 & 0 & 0 \\
0 & 2 & 1 & 0 & 0 & 0 \\
0 & 6 & 6 & 1 & 0 & 0 \\
0 & 24 & 36 & 12 & 1 & 0 \\
0 & 120 & 240 & 120 & 20 & 1
\end{array}\right]=[\mathrm{L}(i, j)]_{6 \times 6},
\end{aligned}
$$

which satisfies (3.4) when $r=5, n=6$.

Remark 3.5. The following identity immediately follows from Theorem 3.4,

$$
L(n, j)=\sum_{k=0}^{n}(-1)^{n-k} s_{r}(n, k) S(k, j ; r) .
$$

Remark 3.6. When $r=0$, the identity in (1.8) can be reduced to

$$
\sum_{j=0}^{n}(-1)^{n-j} L(n, j) L(j, k)=\delta_{n, k},
$$

which yields the following matrix relation

$$
[L(i, j)]_{n \times n}^{-1}=\left[(-1)^{i-j} L(i, j)\right]_{n \times n} .
$$

Multiplying both sides of $(3.4)$ by $[L(i, j)]_{\mathfrak{n} \times \mathfrak{n}}^{-1}$ gives

$$
\left[(-1)^{i-j} L(i, j)\right]_{n \times n}\left[(-1)^{i-j} s_{r}(i, j)\right]_{n \times n}[S(i, j ; r)]_{n \times n}=I_{n}
$$

\section{Concluding remarks and observation}

The two methods of Qi [9] in deriving new explicit formula for Bell numbers have been successfully applied to obtain new explicit formula for r-Bell numbers. However, unlike the one obtained by Qi, these two methods yield two different forms of explicit formulas for $r$-Bell numbers. Moreover, these explicit formulas imply several matrix relations involving $r$-Bell numbers, $r$-Stirling numbers of the first and second kind, the r-Lah numbers, and the ordinary Lah numbers. With these results, one may try to apply Qi's methods to obtain new explicit formulas for other Bell-type numbers like the r-Dowling numbers. 


\section{Acknowledgment}

The authors would like to thank the Center for Research and Development of Cebu Normal University for funding this research project. The authors would also like to thank the referees for their helpful comments and suggestions.

\section{References}

[1] K. N. Boyadzhiev, Lah Numbers, Laguerre Polynomials of Order Negative One, and the $n$th Derivative of $\exp (1 / x)$, Acta Univ. Sapientiae Math., 8 (2016), 22-31. 3.1

[2] A. Z. Broder, The r-Stirling numbers, Discrete Math., 49 (1984), 241-259. 1, 2

[3] L. Comtet, Advanced Combinatorics, D. Reidel Publishing Co., Dordrecht, (1974). 3.1

[4] R. B. Corcino, J. T. Malusay, J. Cillar, G. Rama, O. Silang, I. Tacoloy, Analogies of the Qi formula for some Dowling type numbers, arXiv, 2018 (2018), 22 pages. (Accepted for Publication in Utilitas Mathematica). 2

[5] S. Daboul, J. Mangaldan, M. Z. Spivey, P. J. Taylor, The Lah numbers and the $\mathrm{n}$-th derivative of $\mathrm{e}^{1 / x}$, Math. Mag., 86 (2013), 39-47. 3.1

[6] M. Koutras, Non-Central Stirling Numbers and Some Applications, Discrete Math., 42 (1982), 73-89. 1, 1

[7] I. Mező, The r-Bell numbers, J. Integer Seq., 14 (2011), 14 pages. 1

[8] G. Nyul, G. Rácz, The r-Lah numbers, Discrete Math., 338 (2015), 1660-1666. 1

[9] F. Qi, An explicit formula for the Bell numbers in terms of Lah and Stirling numbers, Mediterr. J. Math., 13 (2016), 2795-2800. 1, 3, 4 